\title{
Téoros
}

Revue de recherche en tourisme

\section{Tourisme et transport au Québec}

\section{Marcel Pouliot et Joanne A. Léveillée}

Volume 14, numéro 2, été 1995

Le tourisme : toute une histoire!

URI : https://id.erudit.org/iderudit/1075100ar

DOI : https://doi.org/10.7202/1075100ar

Aller au sommaire du numéro

Éditeur(s)

Université du Québec à Montréal

ISSN

0712-8657 (imprimé)

1923-2705 (numérique)

Découvrir la revue

Citer cet article

Pouliot, M. \& Léveillée, J. A. (1995). Tourisme et transport au Québec. Téoros, 14(2), 32-35. https://doi.org/10.7202/1075100ar d'utilisation que vous pouvez consulter en ligne.

https://apropos.erudit.org/fr/usagers/politique-dutilisation/ 


\title{
Tourisme et transport au Québec
}

\author{
Marcel Pouliot et Joanne A. Léveillée*
}

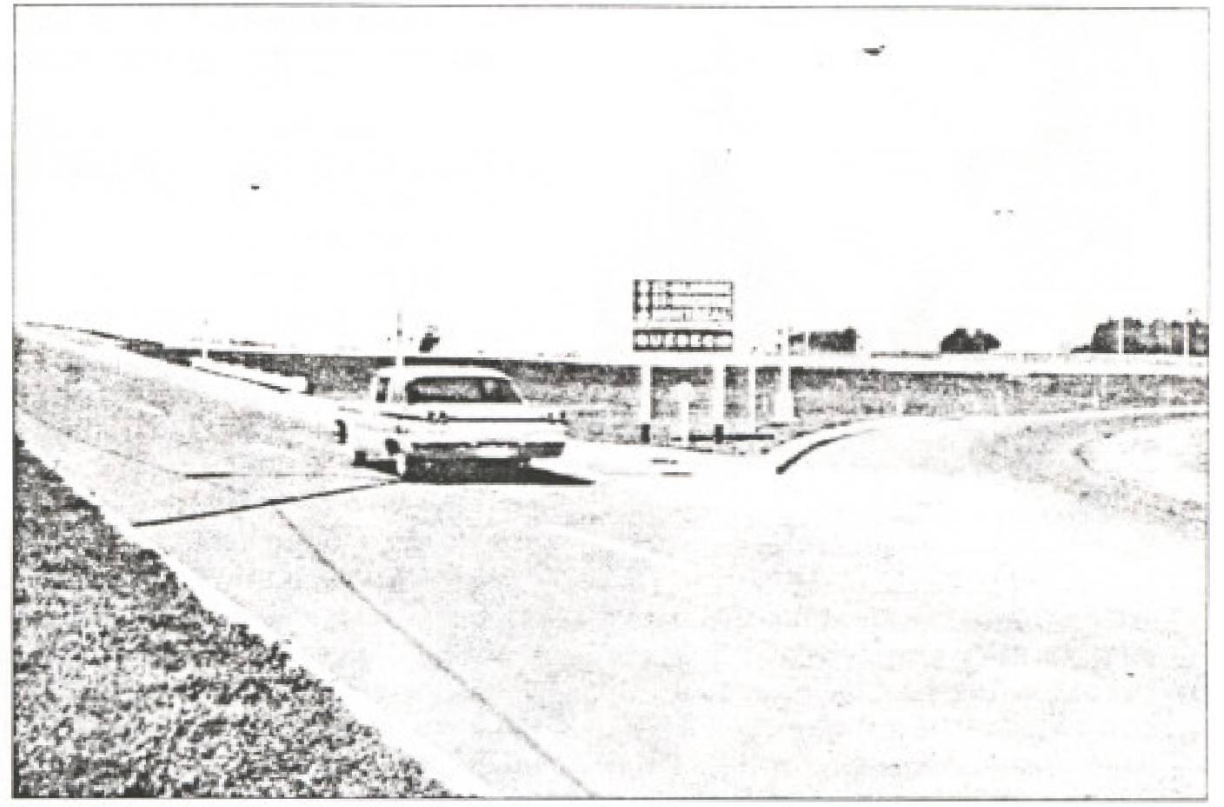

La circulation liée aux loisirs de masse ne commence que vers 1950 et conoerné avant tout, la route. La transcanadienne (ici à l'entrée de Drummondville vers 1960) a relié d'abord la métropole à la capicale du Québec. Photo : Office du film du Québec.

Madame Joannè A, Léveillée est chargè de cours au département de góographie et téledétection à l'Université de Sherbróke. Monsieur Marcel Pouliot est vice-doyen a la recherche a l'Univer sité de Sherbrooke.

Il $y$ a des siècles que les hommes voyagent pour le plaisir. L'Antiquité a certainement eu ses touristes mais l'histoire moderne nousapprend quec'est d'aborden fonction des grands mouvements liés aux pèlerinages que le tourisme débute. Par la suite, la multiplication et la démocratisation des moyens de transport ont généralisé les habitudes touristiques en augmentant l'accessibilité de points ou de régions du globe.

Outre les pèlerinages, le tourisme s'est aussi intéressé aux monuments nés de grandes civilisations et par la suite à des phénomènes plus spécifiques comme la montagne et la mer. Avec les années, il y a eu modifications des finalités touristiques, mais le transport y a constamment joué un rôle.

Capot-Rey (1946) indique en complément qu'on observe de la circulation touristique surtout dans les pays qui sont assurés de générer des ressources financières, un apport que procure uneéconomie bien déve- loppée et qui ont le privilège d'avoir des loisirs. Letourismeest liéauxaturaitsetaux ressources qu'une ville, qu'une région ou qu'un pays peuvent offrir ainsi qu’à la capacité des personnes à se déplacer et à en assumer les coûts.

\section{La situation québêcoise}

Au Québec, les activités touristiques se dont développées depuis plus d'une centaine d'années et se distribuent dans toutes les régions. Le tourisme est historiquement l'affaire de quelques-uns qui s'adonnent à des loisirs de groupe mais aussi aux étrangersqui peuvent se payer les rares modes de transport disponibles pour accéder aux sites les plus intéressants.

Ainsi verra-t-on se combiner des activités touristiques et une série de modes de transport, tous plus performants les uns que les autres, permettant à un public sélectif au départ, mais de plus en plus nombreux par la suite, d'avoir accès à de plus en plus d'endroits. Du bateau à vapeur au chemin de fer, par transport routier ou par trans- 
port aérien, le voyageur attiré par des attraits touristiques contribuera au développement du Québec.

\section{Le transport fluvial et maritime}

Le transport par eau a été le premier mode utilisé au Québec. Il faut dire que la nature a généreusement doté le territoire d'un réseau de lacs et de rivières qui, reliés au fleuve Saint-Laurent, offrent des chemins d'eau largement utilisables. Les grands parcours des commerçants de fourrure furent essenticllement dépendants du transport par eau (a voile, à énergie humaine) pour établir, développer et maintenir la première industrie nationale.

La navigation à voile tant fluviale que côtière, présente des problèmes techniques de navigation et s'avère d'une lenteur désespérante et d'une sécurité relative. D faudra attendre le début des années 1800 pour voir les premiers bateaux à vapeur sur le Saint-Laurent. C'est à un marchand de Montréal, Molson, que revient l'initiative d'avoir vu dans ce mode de transport un outil de commerce hors pair. De 1811 à 1825 , se développera une flotte de bateaux à vapeur assurant la liaison entre Montréal et Québec de même qu'entre les villes et villages qui se situent de part et d'autre du fleuve. Apartir de 1830, le transport fluvial de personnes et de marchandises s'étend à l'ensemble du Saint-Laurent avec l'apparition de quelques autres firmes.

Quelques années auparavant, en 1819 , le transport par petit navire à vapeur s'est étendu sur la rivière Outaouais et a pénétré sur le Saint-Laurenten amont de Montréal et sur les Grands Lacs. Ce double système non encore entièrement relié sera à la base du concept de la construction de canaux qui permettront de joindre dans un tout les opérations de transport effectuées par des naviresà vapeur. En 1825 , le premier canal permettant de passer de Montréal vers la rivière Outaouais et vers Kingston est ouvert. Plus au centre du Québec, le canal Chambly va ouvrir une voie de navigation efficace vers les États-Unis à partir de 1850. Vers la fin des années 1800 , l'élargissement etl'approfondissementdes ouvrages d'art constituent ce qu'on appelle dorénavantlesystème decanauxdes Grands Lacs. Terminés en 1903, cesouvrages vont assurer, entre autres, le passage de navires de plus grande envergure et faire de l'axe Montréal-Grands Lacs, la route commerciale de première importance au Canada.
La voie maritime du Saint-Laurent permertra, trente ans plus tard, aux navires océaniques de pénétrer tout le réseau des Grands Lacset elle sera inaugurée en 1957.

Les liens avec le Saguenay, la Gaspésie et la Côte Nord connaîtront l'époque de la navigation commerciale à voile entre les années 1800 et 1850 . Rapidement, cette dernière sera déclasséect à partir des années 1870 , à l'exception d'activités de cabotage locales, le navire à vapeur prendra la relève rapidement. Le Saint-Laurent sera alors sillonné dans toutes ses parties par dés navires offrant le transport de passagers et de marchandises.

Par contre, les mutations techniques du transport maritime (navires plus spécialisés et gigantesques) contribueront au déclin des navires à petit tonnage et au regroupement des petits ports échelonnés sur les côtes du fleuve Saint-Laurent.

Le transport fluvial et côtier s'avère, au Québec, d'une importance capitale. Sa première fonction a étế l'interconnexion des villes et villages qui s"étendent le long du Saint-Laurent en assurant la mobilité des personnes et des biens situés sur cet axe. Dans un deuxième temps, le système de transport par canal s'est développé rapidement, dû au fait que le Saint-Laurent constituait la porte d"entrée du centre du continent nord-américain.

\section{Le chemin de fer}

Le chemin de fer est vu au Québec et plus tard au Canada comme un élément de progrès et d'essor économique. Ál'aube du $\mathrm{XX}$ siècle, les quelques lignes ferrées qui constitue le réseau se concentrent dans la vallée du Saint-Laurent à l'intérieur du triangle Québec-Montréal-Sherbrooke. $\mathrm{La}$ fonction du chemin de fer est d"assurer le transit des échanges commerciaux canadiens et de la Nouvelle-Angleterre. Dans une moindre mesure, il dessert locslement les industries situées en amont de la ville de Québec, plus spécialement celles des régions de Montréal et de l'Estrie.

La première ligne de chemin de fer du Québec date de 1853 et se situe dans la région de Chambly sous le nom de: *The Stanstead, Shefford and Chambly Railroads. En 1857, elle est reliée avec le * Grand Trunkw (St-Lawrence andAtlantic Railroad).
Après la Confédération, les subventions gouvernementales versées aux entreprises ferroviaires pour la construction des chemins de fers sont d'abord utilisées pour contribuer à l'ouverture et au peuplement de nouveaux territoires de colonisation dans l'arrière-pays de la vallée du Saint-Laurent. Selon Leclerc(1990), dansla secondemoitié du XIX" siècle, le chemin de fer était perçu comme pouvant solutionner tous les maux de la société québécoise. La construction du chemin de fer fut un prétexte pour favoriser l'ouverture du marché urbain aux produits agricoles et forestiers de l'arrièrepays montéalais, de contrer l'émigration de citoyens québécois vers les Etats-Unis, de réduire le nomadisme de certains colons en les établissant sur des terres neuves et de soutenir l'aménagement et l'occupation du territoire.

De façon générale, la hiérarchisation spatiale du développement du réseau ferroviaire québécois indique que la configuration de l'espace québécois a limité l'expansion des lignes transcontinentales à la rive sud du fleuve Saint-Laurent et à la région de l'Outaouais; qu'au-delà de cet espace, le réseau a un rôle essentiellement régional et que Montréal est un noeud de rencontres par lequel transitent la plupart deschemins de fer pan-canadiens (Lederc: 1990).

\section{Le transport routier}

Dans les secteurs à faible densité, le rếseau routier s'est avéré l'infrastructure la plus adéquate pour répondre aux besoins de la colonisation. Les routes ont permis un accèsmoins coûteuxauxsecteursdu Québec en voie de développement, tout en offrant une fleribilité de desserte que ne pouvait réaliser le chemin de fer. Dans la vallée du Saint-Laurent, les bourgs, sous le régime français, sont répartis en ligne entre les agglomérations urbaines. Le réseau routier, au départ simple et linéaire, se densifie par la suite, pour mieux définir les nouveaux emplacements desvillages. Ces derniers jouent des rôles très divers; ils sont à $l_{\mathrm{a}}$ fois un relais et un centre de services. En effet, grâce aux routes, la liaison villecompagne tend à s'affirmer au XVIII et $\mathrm{XXX}^{n}$ siécles. Courville (1990) mentionne ainsi que Montréal et Québec restent des villes tournées vers l'extérieur, mais qu'elles exercent également une influence sur leur région dont elles tirent une grande partiedeleur subsistanceet de leurseffectifs. 
Le réseau routier actuel date de la fin des années 1930 . Hormisle réseau autoroutier, cette période permet d'établir le pattern des grandes routes régionales d'aujourd'hui. Il faudra attendre les années 1950 pour que la technologie amène l'amétioration des surfaces de roulement. Ces nouveaux équipements plus modernes permettront de répondre à la demande croissante des automobilistes désirant circuler avec rapidité et sécurité.

La venue des autoroutes au Québec reste d'influence américaine. Elle est issue en grande partie d'un besoin de décongestionner les voies de circulation existantes et de créer des liens rapides et efficaces entre les principaux points du territoire. La construction des autoroutes du début des années 1960 amorce inévitablement une série d'impacts sur les milieux naturels et humains.

Dans la région de Montréal, en 1959, les autoroutes sont absentes. A cette époque, seul un tronçon de l'autoroute des Laurentides entre Montréal et SaintJérồme et une partie de l'autoroute Mé́tropolitaine étaient alors construits. Ce $n^{\prime}$ 'est que vers la fin des années 1960 qu'apparaissent les premières voies de circulation rapide. Cesautoroutes contribuent au développement touristique des Laurentides en permettant à la population montréalaisedes'y rendre. Dans les années qui vont suivre, le réseau autoroutier n'a cessé de se perfectionner pour désormais relier, au début des années 1980, l'ensemble des régions du Québec (André́: 1994). Depuis, peu de nouvelles autoroutes ont été construites.

\section{Le transport aérien}

Il faut attendre les années 1920 pour voir l'aviation mise à profit au Québec. En

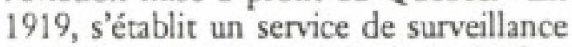
des forêts, d'inventaire forestier et de photographie aérienne de la *Laurentide Air Services: au Lac-à-la-Tortue et quelques années plus tard, cette compagnie s'implante à Roberval. Elle sera suivie de la *Forest Survey Services» et de la * Dominion Aerial Exploration* qui déborderont leur mission d'origine pour effectuer le transport de marchandises et de passagers en lien avec les activités d'exploration et de prospection miniêres en Abitibi et sur la Cote-Nord. En 1924 a lieu le premier vol régulier de passager au Canada par la compagnie *LaurentideAir Services" entre Haileybury, Ont. et Rouyn. Durant la même année, la compagnie Franco-canadienne, sous la direction de Jacques de Lesseps, ouvre la Gaspésie à l'aviation lors des activités intenses de photographies aériennes. Ainsi, tout le sud du Québec bourdonne à la fin des années 1930 d'activités aériennes; les bases de l'aviation moderne sont en quelque sorte jetées.

Le nord québécois n'est pas laissé pour compte. Dès 1927, le gouvernement du Canada implante à partir de Kangiqsujuaq, de l'ille Nottingham et de Killiniq sur le détroitd'Hudson, despatrouillesaériennes chargées de surveiller le mouvement des glaces. La baie d'Ungava est jointe en 1930 à partir de Roberval et de Nitchequon. Les rives orientales de la Baie d'Hudson sont également explorées par la voie des airs au cours des mêmes années à partir du nord de l'Ontario.

Dans le sud du Québec, on remarque une consolidation de petites firmes d'aviation, entre autres, la compagnie *Canadian Airways». En 1927, cette firme assurera le service postal sur la Côte-Nord et Roméo Vachon $y$ créera sa légende. Des centres d'activités aériennes importants pour l'époquesontcréésà Rouyn, Chibougamau, Roberval et St-Félicien.

Pourtant, les années 1934-1936 vont marquer le déclin des activités aériennes commerciales au Québec, l'effort de guerre est imminent et toutes les énergies sont dorénavant mises à $\mathrm{la}$ formation et à la production des personnels et des infrastructures qui constitueront l'assise du développement militaire. Les années de guerre 1939-1945 auront un effet direct sur le développement du transport aérien en dotant le Québec d'une infrastructure qui lui manquait jusqu'alors. La construction d'aéroports méridionaux et nordiques constituera la base du réseau des années 1950 et 1960 .

Au niveau canadien, *Trans Canada Airlines* et *Canadian Pacific Airlines* constituent deux entreprises concurrentes. La première formée en 1937 par le gouvernement canadien aura une mission de relier les capitales des provinces d'un océan à l'autre. Del'autre cốté, la CPAs'est formée dès 1940 par l'amalgamation de 9 petites firmes aériennes autour de la *Canadian Airwaysw et de la wQuebec Airwayss. Afin de régir la concurrence, le gouvernement canadien limitera les actuvités de l'une et de l'autre en les cantonnant dans des territoires respectifs.

Par la suite, les transporteurs commerciaux régionaux font leur apparition. Dès le milieu des années 1950, ces transporteurs, suite à des fusions de petites compagnies de brousse viendront constituer l'ossature provinciale et ouvrir de façon généralisée toutes les provinces au transport aérien. Le Québec dispose de deux de ces transporteurs. *Nordair Ltée s'installe à Montréal et dessert l'ouest et le nord québécois et la compagnie *Québecair», née à Rimouski, s'installe à Montréal et dessert principalement l'est du Québec.

À partir du milieu des années 1950, toutes les régions du Québec sont dorénavant accessibles par le transport aćrien régulier en partant de Montréal ou de Québec alors que les petits transporteurs de brousse continuent à travailler à une échelle plus réduite, principalement vers des secteurs non équipés d'aéroports de moyenneou de grande envergure. Cette cohabitation, de ce qu'on appelle les troisniveauxd'aviation commercale, le national, régional et local se poursuivra jusqu'aux années 1980 où la dếréglementation viendra commeélếment réorganisateur du transport aérien nordaméricain.

\section{Le rốle du transport}

On est souvent porté à se questionner sur le rôle exact que le transport joue ou prend dansl'ensemble des activités humaines. $\mathrm{La}$ ré́ponse la plus explicite vient de Cooley (1894) quand il dit: *Le transport est un moyen par lequel chaque composante de la société est mise en interrelation et grâce auquel la spécialisation et l'organisation sont rendues possiblesw.

Ce point de vue nous fait très bien voir le type de relation qui existe entre toute forme d'entreprise humaine et le transport. Le rôle du transport en est un d'assurer la mobilité des biens et des personnes, de garantir leur accès à des lieux spécifiques et ainsi de contribuer à ce que nous pouvons appeler le développement socio-économiqueetorganisationnel. Dconstitue donc un élément structurant tout en s'avếrant essentiellement un outil, un intermédiaire permettant de relier une demande à un besoin. La multiplication de ces situations crée la circulation, elle-même le reflet d'échanges de toute nature dans un cadre d'économie libérale. Ainsi, le transport 
n'existe-t-il pas pour lui-même, mais est toujours fonction d'impératifs qui lui sont extrinsèques. Somme toute, on ne transporte pasou on ne circule pas pour le plaisir detransporter ou de circuler; on le fait pour une finalité plus fondamentale, reliée de prèsau développement des personnes et de la société dans laquelle tout individu évolue.

Dans ce cadre spécifique, le bref historique des transports au Québec démontre que celui-ci a été implanté en fonction d'impératifsessentiellementéconomiques, dans le but de mettre sur pied une économie de marché. D'un système basé sur l'exploitation des ressources naturelles, au maintien d'une économie rurale semiautarcique, au développement industriel local et nord-américain de plus grande enverguré, le réseau de transportau Québec n'aura été qu'un outil facilitant de façon prioritaire, l'atteinte d'objectifs industriels et commerciaux.

A notre avis, ce n'est qu'après la Seconde Guerre mondiale quele réseaude transport trouve d'autres finalités dont la circulation liée aux loisirs de masse. La prospérité d'aprè̀-guerre aidant, l'explosion démographique s'avérant également un facteur, les changernents dans les habitudes de vie des Québécois bouleversent la mission unique du réseau du transport. A ce titre, le réseau routier s'avère le grand dénominateur commun des déplacements des Québécois; ceux-ci habitués à entrevoir quelques touristes fréquenter en exclusivité certains lieux privilégiés, voudront à leur tour accéder aux plaisirs touristiques de circuler, de se reposer et de profiter des attraits du territoire. Le réseau autoroutier mis en place dans les années 1960 sera la véritable clé de la multiplication des déplacements de loisirs. L'aventure touristique, qui avait jusque là échappée à la plupart des Québécois, est dorénavant à portée de route.

\section{Conclusion}

Quels liens peut-on établir entre le tourisme et le transport? La réponse demeure complexe car le transport s'avère un instrument de la mobilité des personnes et des biens et à ce titre il devient ce que d'uns appelleront un élément essentiel et ce que d'autres nommeront un élément stratégique. Dans le cas du Québec, il est évident que le transport a été au début du développement économique et social et non un moyen facilitant l'activité touristique pour la bonne raison que le tourisme n'existait à peu prè̀s pas.

Les premiers et véritables touristes au Québec constituaient une elite très exclusive qui fréquentait pour son plaisir des sites d'intérêt qu'on peut identifier à quelques lieux spécifiques pour nenommer que Murray Bay, Metis Beach, North Hatley, Morin Heights et les nombreux clubs privés de pêche le long des rivières de la Côte-Nord et de la Gaspésie. Le transport par bateau, train ou avion de brousse leur était alors réservé, et noblesse et richesse obligeant, les raisons sociales destransporteurs étaient de langue anglaise.

Il faudra attendre pour constater une contrepartie francophone; phénomène unique, les premiers mouvements touristiques de groupe avaient pour destination la trilogie Oratoire Saint-Joseph, Cap-dela-Madeleine et Sainte-Anne-de-Beaupré. Autres temps, autres moeurs. Le transport ferroviaire et surtout la route auront étéles modes privilégiés pour accéder à ces sites.

Dans l'évolution des réseaux de transport et des activités touristiques, la révolution tranquille des anníes 1960 agira comme grande niveleuse des différences culturelles; l'élargissement de la richesse collective, des conditions de travail plus favorables de mềne qu'un réveil face aux autres sociétés, contribueront à la vocation touristique des Québécois. Cette soif et cet intérêt pour les voyages, sans doute motivés par le besoin de s'affranchir de ses propres réalités quotidiennes, dont un hiver interminable, constituent la véritable base de l'explosion touristique avant même toutes activités liées au transport.

D'un autre côté́, le Québec développera un produit touristique largement accessible aux étrangers misant sur un milieu naturel et des traits culturels uniques; soulignons, dans un contexte très contemporain, l'existence de produits basés sur des randonnées hivernales en motoneige ou en traineaux à chiens, des circuits en canot ou en kayaks de mer, des croisières mettant en évidence l'observation des baleines. Toutes ces activités comme la redécouverte des rives Saint-Laurent par bateau illustrent fort bien le lien qui existe entre le tourisme et le transport. Le tourisme peut avoir une existence propre, mais dépend du transport pour prendre son véritable sens et pour see réaliser. Le transport n'a aucune raison d'exister si cen'est que des'ayérer au service du premier. Fondamentalement, le transport est l'outil de la mobilité (des gens et des choses) et une condition sine qua non qui accompagne toute forme de développement économique et social dont le tourisme forme un élément. $f$

\section{BIBLIOGRAPHIE}

ANDRE, Francois, Les effete dune autoroute sur t localisation des activité commerciales ot de cervices, rapport d'étape, mémoire de mahtise, Institut d'urbanisme, Univer itée de Montréal, 1994, $76 \mathrm{p}$.

BOOTH, J. Derek, Railwaye of Souther n Quebee, wol. 1. A Railfare Book, $1982,160 \mathrm{p}$.

CAPOT-AEY, Robert, Gbogr aphie de la circulation sur les continents. Editions Gallimard, collection Geographie humaine, 1946, $296 \mathrm{p}$.

COURVILLE, Serge, Entre ville et campagne, f'ensor du village dans les seigneuries du Bas-Canada. Ed̆ioñs PUL, 1990, 335 p.

COOLEY, Charles $\mathrm{H}_{\text {., }}$, The Theory of Transportation, in Journal of The Amarican Economic Association. vol. 9, no 3, 1894 .

GLAZEBROOK G.P. de T. A Histor Y of Transportation in Canada, vol. 1 and wol. 2. MeClelland \& Steward Ltd. 1967.

LASSERRE, Jean-Claude, Les ports qubbecois necesith dune relance concert 6 e, CRT, Université de Montreal, publication no 176, collection Notes et documents no 80-07, 1980, $84 \mathrm{p}$.

LASSERAE, Jean-Claude, Le Saint-Laurent, grande porte de I'Amerique, Éditions Hurubise $\mathrm{HMH}_{\text {. }}$ Cahiers du Québec, collection Geographie, 1980. $753 \mathrm{p}$.

LECLERC, Richard, La politique ferroviaire qubbecoise et Forganisation du territoire [1867-1990]. thetse (Ph.D.). Faculte des Lettres, departement de geographie, Universite Laval, 1991,613 p.

MAIN, JAK Kes voyageurs de l'air, Imprimeur de la Reine, Ottawa, 1967, 433 p.

POULIOT, Marcel, Gtographiedes transports ariens au Nouveau-Qubbec, memoire de maltrise, Universine Laval, $1971,206 \mathrm{p}$.

AIVEST, Jacques, Non aviateuri, det wout de Falia auxpilotes delignes, Edisons del'Homme, 1981 , $274 \mathrm{p}$

WACKERMANN, Gabriel, Tourisme et transport, Editions SEDES, Pans, 1993, 279 p. 